\title{
Effects of Bitter Melon Saponin on the Glucose and Lipid Metabolism in HepG2 Cell and C. elegans
}

\author{
Juan Bai, ${ }^{1}$ Ying Zhu, ${ }^{1}$ Jie Li $\mathbb{D},{ }^{1}$ Yi Zhang, ${ }^{1}$ Ying Dong, ${ }^{1}$ and Xiang Xiao $\mathbb{D}{ }^{1,2}$ \\ ${ }^{1}$ School of Food and Biological Engineering, Jiangsu University, Zhenjiang 212013, China \\ ${ }^{2}$ Jiangsu Jiangnan Biotechnology Co. Ltd., Zhenjiang 212013, China \\ Correspondence should be addressed to Xiang Xiao; xiaoxiang1@aliyun.com
}

Received 20 May 2020; Revised 30 May 2020; Accepted 5 June 2020; Published 24 June 2020

Academic Editor: Quancai Sun

Copyright $(2020$ Juan Bai et al. This is an open access article distributed under the Creative Commons Attribution License, which permits unrestricted use, distribution, and reproduction in any medium, provided the original work is properly cited.

\begin{abstract}
This study tried to explore how saponins from bitter melon (BMS) affect the glucose and lipid metabolism in palmitic acid-treated HepG2 cell and glucose-treated Caenorhabditis elegans (C. elegans). Results showed that BMS could effectively accelerate glucose consumption and elevate the levels of glycogen and ATP in palmitic acid-treated HepG2 cell, while significantly decreasing the triglyceride (TG) content. qRT-PCR data indicated that BMS might promote fatty acid $\beta$-oxidation by AMPK-ACC2-CPT1 pathway and glucose uptake by upregulating GLUT4 expression. In the model of glucose-treated C. elegans, we observed that BMS obviously inhibited fat accumulation, along with no toxicity towards some physical activities. The potential mechanism of BMS in the metabolism involved the suppression of synthesis of polyunsaturated fatty acids and enhancement of fatty acid $\beta$-oxidation. Taken together, BMS exhibited ability of regulating energy metabolism in HepG2 cell line and C. elegans.
\end{abstract}

\section{Introduction}

In the last few decades, obesity and its related metabolic disorders have emerged as public health issues worldwide [1]. Obesity is developed with the evidence of body weight gain, insulin resistance, hyperlipaemia, and so on, increasing the risk of II type diabetes, nonalcoholic fatty liver disease, and some other metabolic syndromes [2]. Nowadays, much attention has been paid to some natural nutrients to early intervene the obesity. Bitter melon (Momordica charantia L.) is commonly used as a medicinal and edible plant for disease prevention, particularly obesity and diabetes [3]. Saponin is one of the main active components in bitter melon, whose structure consists of triterpenes and steroids [4]. Large amounts of mechanism studies have shown that the saponins of bitter gourd have physiological activities such as lowering blood glucose and improving insulin resistance, which are recognized as insulin-like $[5,6]$. What is more, the fat-lowering effect of BMS is another bioactivity, which interacts with the glucose metabolism to improve the insulin resistance. In vitro study suggested bitter melon triterpenoid reduced preadipocyte viability and lipid accumulation by downregulating PPAR $\gamma$ in 3T3-L1 cells [7]; in vivo study reported that saponin extract from bitter melon significantly suppressed body weight gain and visceral fat accumulation in the PPAR $\alpha$ - and PPAR $\gamma$-mediated pathways in obese mice [8].

Caenorhabditis elegans (C. elegans, also referred to as 'nematode' hereinafter), is a whole-system organism for the evaluation and mechanism study of toxicity or bioactivity. Recently, C. elegans has become an excellent model for studying the mechanism of lipid metabolism due to its high homology to mammals and rich genes-deficient mutants [9]. Lipid metabolism includes fatty acid synthesis, oxidation, unsaturation, and elongation, which are deeply evolutionarily conserved in C. elegans to the mammals. Additionally, in the obese, liver is one of the important insulin-sensitive tissues and HepG2 cell line is commonly applied to study the lipid metabolism in hepatic function by stimulation with fatty acids.

Therefore, this study aimed to figure out the impacts of BMS on ameliorating insulin sensitivity and fat deposition in hepatic cell. Based on this, we further confirm the fatlowering effects of BMS in C. elegans and preliminarily 
reveal the potential mechanism in the models we used in this current study.

\section{Materials and Methods}

2.1. Materials and Chemicals. Fresh bitter melons were collected from Lvjian Agricultural Station (Yangzhong City, China) and authenticated by Jiangsu Academy of Agricultural Science. HepG2 cell was purchased from Shanghai institute of biochemistry and cell biology. Escherichia coli OP50 and N2 strain of C. elegans was obtained from Caenorhabditis Genetics Center, University of Minnesota, USA. Fetal bovine serum and Dulbecco's modified Eagle's medium (DMEM) were purchased from GIBCO Company, USA. Metformin and palmitic acid were bought from Sigma, USA. MTT assay, Oil Red O staining assay, glucose and glycogen contents determination, ATP and TG contents determination, and protein assay kits were purchased from Nanjing Jiancheng Bioengineering Institute, China. TaKaRa MiniBEST Universal RNA extraction kit, PrimeScript ${ }^{\mathrm{TM}}$ RT Master Mix kit, and SYBR Premix Ex $\mathrm{Taq}^{\mathrm{TM}}$ kit were purchased from Takara Bio Company, Japan. All primers were purchased from Sangon Biotech Company, China. All of the other reagents and solvents were purchased from Sinopharm Chemical Reagent Co. Ltd., China and were of either analytical or chromatographic grade.

2.2. BMS Preparation. Unripe bitter melons were washed thoroughly in water and the seeds were removed. Then, the pulp was thinly sliced, freeze-dried, and milled (diameter $<100 \mu \mathrm{m}$ ). Bitter melon powder was added with $75 \%$ ethanol for reflux extraction twice at $80^{\circ} \mathrm{C}$, and the filtrate was collected. Then, n-butanol saturated with water was applied to extract from the filtrate for 3 times until the concentrated n-butanol phase was brown and sticky. Methanol and acetone were added to produce precipitation, which then was frozen as the BMS.

2.3. HepG2 Cell Culture and HepG2-IR Cell Model. The cells were cultured in monolayers up to $80 \%$ confluence in DMEM supplemented with $10 \%$ heat-inactivated fetal calf serum and $1 \%$ penicillin/streptomycin at $37^{\circ} \mathrm{C}$ in a humidified incubator supplied with $5 \% \mathrm{CO}_{2}$. Cells in the logarithmic growth phase were used for all the studies described below.

For model of insulin resistance (IR), the supernatant of HepG2 cells inoculated in 96 plates was discarded and replaced with serum-free DMEM medium for 12 hours to keep fasting. Then, the supernatant was discarded, washed twice with PBS, and added with serum-free culture medium containing palmitic acid (the final concentration was $0.25 \mathrm{mM}$ ) for 12 hours to induce HepG2-IR cell model.

2.4. Cell Viability and Glucose Consumption Determination. Based on the establishment of HepG2-IR cell model, BMS at different concentrations of 50, 100, 250, 500, 750, 1000, and $2000 \mu \mathrm{g} / \mathrm{L}$ were incubated together with palmitic acid after $12 \mathrm{~h}$ fasting. The cell viability was detected by MTT method [10].

For glucose consumption determination, groups were divided into blank group (without cell), normal group, IR model group, and IR model groups with BMS or $2 \mathrm{mM}$ metformin. After $12 \mathrm{~h}$ treatment together with palmitic acid, the glucose content determination kit and MTT assay kit were used to measure the glucose consumption, calculated by the following formula:

$$
\text { relative glucose consumption }(\%)=\frac{\text { (glucose contents of blank group-IR model groups)/cell viability of IR model groups }}{\text { (glucose contents of blank group }- \text { normal group)/cell viability of normal group }} \times 100 \% \text {. }
$$

2.5. Glycogen, TG, and ATP Contents Determination for Cells. For glycogen, TG, and ATP contents determination, groups were divided into normal group, IR model group, IR model groups with BMS or $2 \mathrm{mM}$ metformin. After $12 \mathrm{~h}$ treatment together with palmitic acid, the kits for glycogen, TG, ATP, and protein contents determination were applied. Formulas were as the following:

$$
\begin{aligned}
& \text { relative glycogen content }(\%)=\frac{(\text { glycogen content of IR model groups }) / \text { protein content of IR model groups }}{(\text { glycogen content of normal group }) / \text { protein content of normal group }} \times 100 \%, \\
& \text { relative TG content }(\%)=\frac{(\text { TG content of IR model groups }) / \text { protein content of IR model groups }}{(\text { TG content of normal group }) / \text { protein content of normal group }} \times 100 \%, \\
& \text { relative ATP content }(\%)=\frac{(\text { ATP content of IR model groups }) / \text { protein content of IR model groups }}{(\text { ATP content of normal group }) / \text { protein content of normal group }} \times 100 \%
\end{aligned}
$$


2.6. C. elegans Culture. M9 buffer, S-complete, and nematode growth medium (NGM) used for C. elegans culture were prepared as previously described [11]. Glucose plates were prepared by adding glucose $(1 \mathrm{M}$, sterile filtered) into the autoclaved NGM after cooling. Worms were synchronized according to standard protocols [12]. For maintenance, worms were grown on NGM plates and E. coli OP50 was freshly provided as the food source. For assays, $C$. elegans were treated by $\mathrm{ddH}_{2} \mathrm{O}$ or BMS for $48 \mathrm{~h}$ from L1 to L4 in the NGM with or without glucose (1 mM final concentration). Groups were divided to normal, model, and model with BMS 100 and $250 \mu \mathrm{g} / \mathrm{L}$.

2.7. Oil Red O Staining for C. elegans. Synchronized L1 worms were treated with BMS (100 or $250 \mu \mathrm{g} / \mathrm{mL})$ for $48 \mathrm{~h}$ to late L4 stage followed by Oil Red O staining. Oil Red O staining ( 1000 worms per each group) was conducted by washing animals with $1 \mathrm{x}$ phosphate-buffered saline (PBS). Worms were washed with 1x PBS three times, fixed with $4 \%$ formaldehyde for $15 \mathrm{~min}$ and dehydrated with $60 \%$ isopropanol for $15 \mathrm{~min}$. Oil Red O solution $(0.5 \mathrm{~g} / 100 \mathrm{~mL}$ in isopropanol) was diluted in double distilled water to $60 \%$ working solution and filtered. Fixed worms were incubated in the working solution overnight at room temperature. Dye was removed after allowing worms to settle, and $200 \mu \mathrm{L}$ of $1 \mathrm{x}$ PBS $0.01 \%$ Triton X-100 was added. 20-30 Oil Red O stained worms were randomly selected for imaging with a Leica microscope outfitted with DIC optics (Leica, Wetzlar, Germany) under identical settings and exposure times.

2.8. TG Assay for C. elegans. Synchronized L1 worms were treated with BMS ( 100 or $250 \mu \mathrm{g} / \mathrm{mL})$ for $48 \mathrm{~h}$ to late L4 stage followed by TG measurement. C. elegans ( 2000 worms per each group) were broken by an ultrasonic cell disrupter and centrifuged to obtain the supernatant. The supernatant was reacted with the kit and the absorbance was measured at a wavelength of $510 \mathrm{~nm}$. The TG and the protein assays were conducted according to the manufacturer's instructions. TG content was normalized with protein concentration determined by Bradford assay.

2.9. Locomotive Activity, Brood Size, and Lifespan Assay. For head thrashing, worms were washed with M9 buffer and dropped on another NGM plate. The head thrashing frequency ( 30 worms in each group) was observed under a stereo microscope as a change in the direction of bending at the midbody and the number of times was recorded in one minute. The experiment was repeated at least 3 times.

For body bend assay, the examined nematodes $(\sim 30$ worms in each group) were picked onto another NGM plate and scored for the times of bodies bends in an interval of $20 \mathrm{sec}$. A body bend was counted as a change in the direction of the part of nematodes corresponding to the posterior bulb of the pharynx along the $y$-axis, assuming that nematode was moving along the $x$-axis. The experiment was repeated at least 3 times.
For brood size, 1 random worm in each group was transferred to a new NGM plate with OP 50, and each group has three duplicates. Worms were transferred to fresh NGM plates during the reproduction period, and the eggs left were allowed to hatch and grow to L4 stage before counting the number of progeny of each worm.

For lifespan, about 100 worms in each group were transferred every day to the corresponding fresh NGM plates from L4 stage until all died. The number of surviving worms were counted and the actual number in the statistical results varies slightly due to the loss of dead or censored animals. Indicators of death included lack of movement, the stress movement of the parasite, and a lack of pharynx contraction after one or two attempts at gentle touching.

2.10. Quantitative Real-Time PCR ( $q R T-P C R$ ) for Cells and C. elegans. RNA of HepG2 cell and C. elegans samples were extracted by the TaKaRa MiniBEST Universal RNA Extraction Kit. Prime Script ${ }^{\mathrm{TM}}$ RT Master Mix Kit was applied to synthesis cDNA according to the manufacturer's protocol. cDNA was amplified and quantified in a CFX96-PCR detection system using the SYBR Premix Ex Taq II Kit. Primer sequences are listed in Table 1. Data were normalized to GAPDH gene in cells and $\beta$-actin gene in C. elegans, analyzed using the $\triangle \triangle \mathrm{Ct}$ method [13].

2.11. Statistical Analysis. Data are presented as means $\pm S E$ and analyzed with the Statistical Analysis System (SAS Institute, NC, USA). Statistical analysis was performed using one-way analysis of variance, followed by Tukey's multiple range test to compare between groups. The significance of differences was defined at the $p<0.05$ level.

\section{Results and Discussion}

3.1. Influences of BMS on the Cell Viability and Glucose Consumption. Figure 1(a) showed the effect of BMS at different concentrations on the survival rate of HepG2 cells for $12 \mathrm{~h}$. There were no significant effects of BMS concentration from $50 \mu \mathrm{g} / \mathrm{mL}$ to $250 \mu \mathrm{g} / \mathrm{mL}$ on cell viability $(p>0.05)$, while the concentrations of BMS at 500, 750, 1000 , and $2000 \mu \mathrm{g} / \mathrm{mL}$ were obvious $(p<0.05)$, indicating that BMS concentration greater than $500 \mu \mathrm{g} / \mathrm{mL}$ had a significant inhibitory effect on the cell growth. Therefore, the concentration range of BMSE was determined to be between 50 and $250 \mu \mathrm{g} / \mathrm{mL}$.

The effects of BMS at different concentrations on glucose consumption in HepG2 cells were shown in Figure 1(b). With the palmitic acid induction, the glucose consumption of cells in the model group was significantly lower than that in the normal group $(p<0.05)$, indicating that the HepG2IR cell model was successfully established. Similar to the effect of Met, the BMS concentration at 100 and $250 \mu \mathrm{g} / \mathrm{mL}$, not $50 \mu \mathrm{g} / \mathrm{mL}$, obviously enhanced the glucose consumption of HepG2 insulin resistant cells ( $p<0.05$ ), which was largely due to the stimulation of GLUT4 transferring to the membrane [14]. 
TABle 1: Primer sequences for cells and C. elegans.

\begin{tabular}{lccc}
\hline Models & Genes & Forward sequences $\left(5^{\prime}-3^{\prime}\right)$ & Reverse sequences $\left(5^{\prime}-3^{\prime}\right)$ \\
\hline & AMPK & ACAGGCATATGGTGGTCCATAGAGA & TTGGGTGAGCCACAACTTGTTC \\
ACC2 & GCAAGAACGTGTGGGGTTACT & TCGTAGTGGGCTTGCTGAAAA \\
& CPT1 & GGTGAACAGCAACTATTATGTC & ATCCTCTGGAACTGCATC \\
& GLUT4 & GCTACCTCTACATCATCCATCTC & ACCCGGCTACAAAGACC \\
& PGC-1 $\alpha$ & GGGGCTCTCCAGAACATCAT & TCAAGGGGTCTACATGGCAA \\
& GAPDH & GGGGCTCTCCAGAACATCAT & TCAAGGGGTCTACATGGCAA \\
\hline \multirow{4}{*}{ Felegans } & Fat-5 & CGGCCGCCCTCTTCCGTTAC & TGGCTGCCATCCGACCCAGT \\
& Fat-6 & TCAACAGCGCTGCTCACTAT & TTCGACTGGGGTAATTGAGG \\
& Fhr-49 & CAACAGCGCTGCTCACTATT & CACCAACGGCTACAACTGTG \\
& $\beta$-Actin & AGGCTCGTGTCAATCAAGAGATGTG & ATGCCGATGCTCCAGAATCACTTC \\
& & GCCGGAGACGACGCTCCACGCG & GCCTCGTCTCCGACGTACGAGTC
\end{tabular}

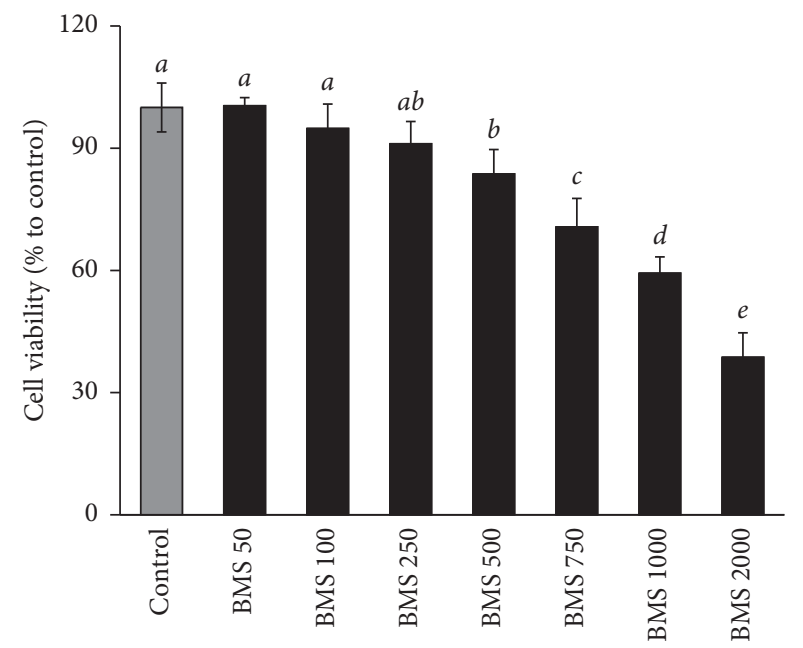

(a)

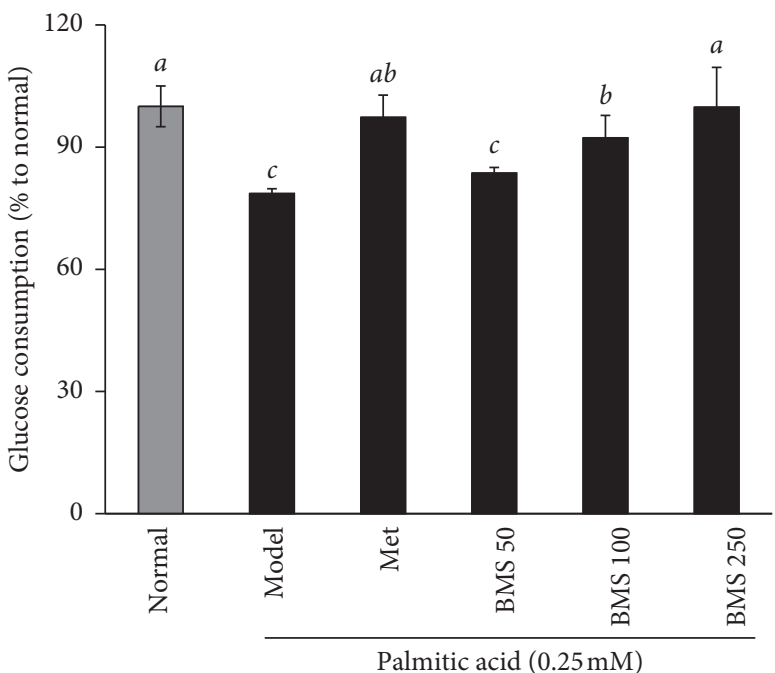

(b)

FIGURE 1: Effects of different concentrations of BMS on the survival rate (a) and glucose consumption (b) of HepG2 cells. Values are expressed as means \pm SE. Statistical analysis was performed using ANOVA. Different superscripts were considered significantly different. Met: metformin; BMS: bitter melon saponins.

3.2. BMS Altered the Levels of Glycogen, TG, and ATP in Insulin-Resistance HepG2 Cell. The liver is the main part of the body that regulates blood glucose. As shown in Figure 2(a), the glycogen content in model group was significantly decreased compared to the normal group $(p<0.05)$, suggesting that the glucose metabolism, especially the gluconeogenesis, was disordered after palmitic acid induction. However, BMS at 100 and $250 \mu \mathrm{g} / \mathrm{mL}$ remarkably normalized the glycogen level of HepG2-IR cells compared with model group, as well as the Met $(p<0.05)$. Consistent with the results from Min et al. found that triterpenoids isolated from bitter melon could inhibit gluconeogenesis in both L6 myotubes and 3T3-L1 adipocytes to decrease the glucose level [15].

Long-term treatment with palmitic acid in HepG2 cells accelerated the fat accumulation, characterized by the increase of TG level, as shown in Figure 2(b). Supplementation with BMS at 100 and $250 \mu \mathrm{g} / \mathrm{mL}$ concentrations effectively decreased the TG content compared to the model group, suggesting BMS could inhibit the fat deposition of HepG2IR cells $(p<0.05)$. In 3T3-L1 cell line, it was reported that bitter melon triterpenoid extract could reduce preadipocyte viability and lipid accumulation [7].

ATP level reflects the status of energy metabolism. Palmitic acid can lead to the uncoupling of mitochondrial respiratory chain of cells and reduce the ATP level, causing the idling state of oxidative phosphorylation [16]. Compared with the normal group, the ATP content in the model group was strikingly declined $(p<0.05)$. Only BMS at $250 \mu \mathrm{g} / \mathrm{mL}$ exhibited improved ATP level, even not the Met, which illustrated that BMS not only recovered the levels of glycogen and TG, but also improved the disorder of energy metabolism. Metformin failed to increase ATP content, probably due to its abilities to inhibit mitochondrial respiratory chains, therefore reducing ATP production and declining the ratio of ATP/AMP, which in turn activates AMPK [17].

3.3. Effects of BMS on the Expressions of Glucose and Lipid Metabolism Related Genes in HepG2-IR Cells. To discover the potential mechanism of BMS ameliorating the indexes of metabolism, we applied qRT-PCR to check the expressions of related genes. From Figure 3(a), for genes related fatty 


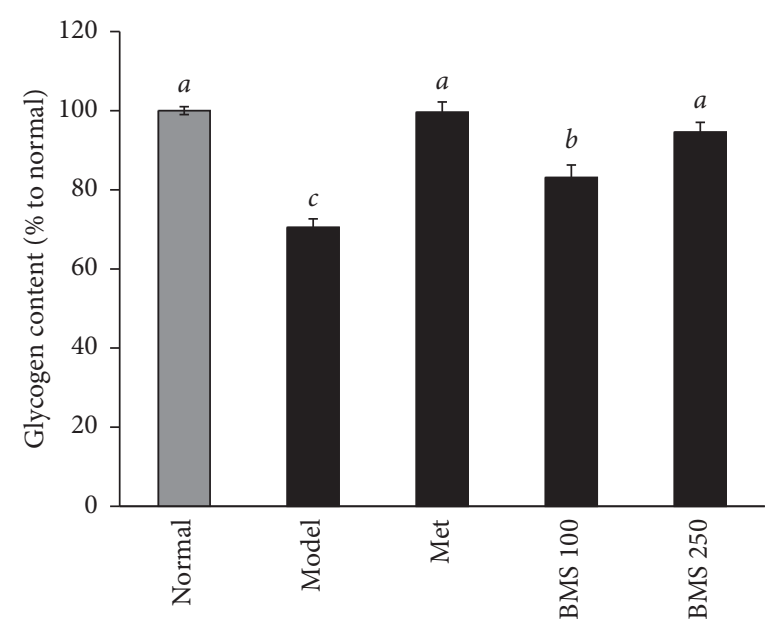

Palmitic acid $(0.25 \mathrm{mM})$

(a)

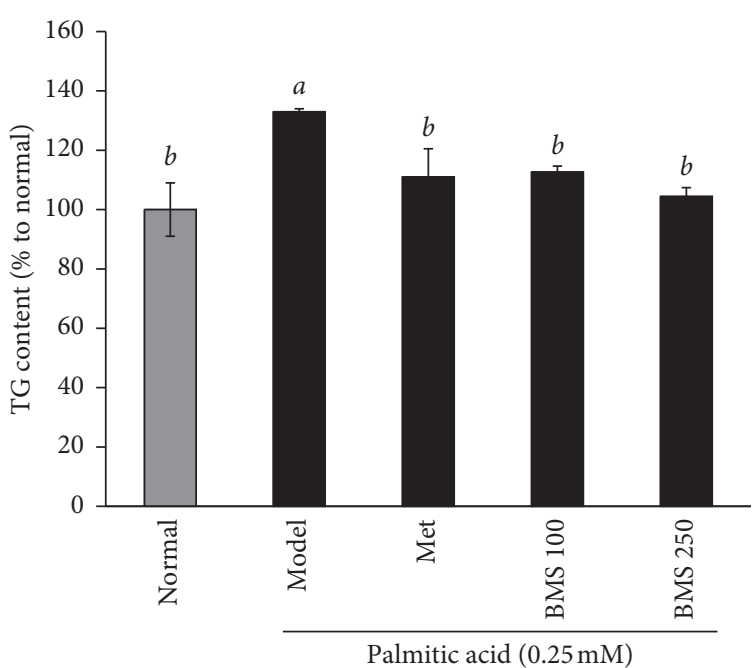

(b)

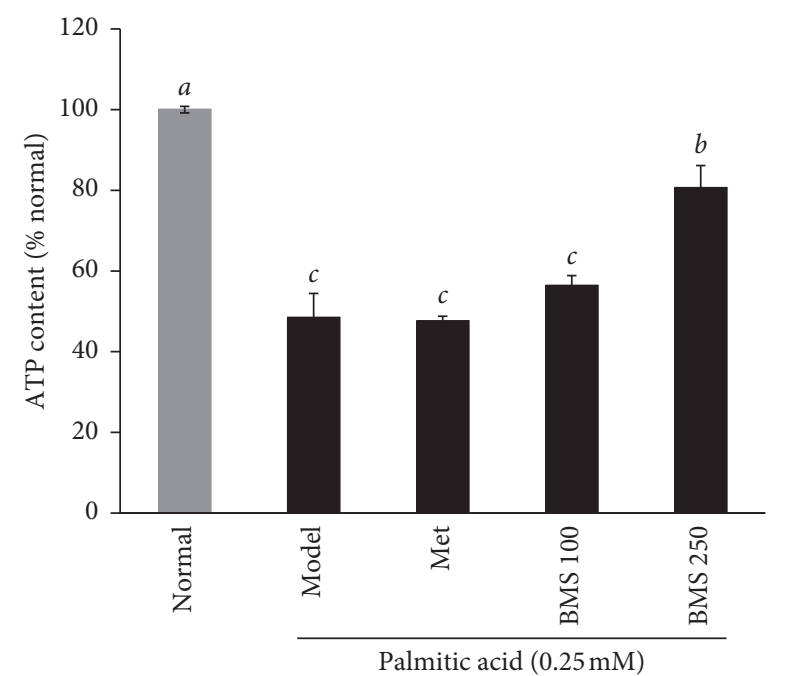

(c)

Figure 2: Effect of BMS on the levels of glycogen (a), TG (b), and ATP (c) of HepG2-IR cells. Values are expressed as means \pm SE. Statistical analysis was performed using ANOVA. Different superscripts were considered significantly different. HepG2-IR cells: HepG2 insulin resistance cells; TG: triglyceride; Met: metformin; BMS: bitter melon saponins.

acid $\beta$-oxidation, BMS at $250 \mu \mathrm{g} / \mathrm{mL}$ concentration largely stimulated the mRNA expression of AMPK and CPT1, while decreasing the ACC2 mRNA level $(p<0.05)$, which enhanced fatty acid $\beta$-oxidation [18]. For genes related glucose intake, BMS could augment the expressions of PGC- $1 \alpha$ and GLUT4, similar to the effect of Met. Increased GLUT4 translocation from the cytoplasm to the membrane promotes glucose uptake [19]. In line with Han's study, triterpenoids in bitter melon improved glucose homeostasis by upregulating GLUT4 in streptozotocin-induced diabetic mice [20]. Based on these results, we speculated the potential pathways of BMS involving in the metabolism included the fatty acid $\beta$-oxidation and glucose intake (Figure 3(b)).

\subsection{BMS Altered the Fat Accumulation of Glucose-Treated C.} elegans. C. elegans has been widely used as an in vivo model for exploring the genetic regulation of fat storage, due to the fact that many aspects of fat synthesis and breakdown pathways characterized in humans are conserved in this easy-handling organism [21]. The intestine in C. elegans consists of 20 cells and is responsible for food digestion and nutrient absorption as well as the synthesis and storage of fat [22]. As BMS exhibited strong effects on the lipid metabolism in vitro, we further assessed the impact of BMS on the overall fat and lipid metabolism in C. elegans by Oil Red $\mathrm{O}$ staining and TG assay to confirm it. Figure 4 indicated that $1 \mathrm{mM}$ glucose could dramatically stimulate the fat accumulation in $C$. elegans compared to the normal group, while BMS at both 100 and $250 \mu \mathrm{g} / \mathrm{mL}$ concentrations obviously decreased the overall fat in the body of $C$. elegans $(p<0.05)$. Likewise, a striking reduction in TG level was observed in BMS groups than the model group, which further confirmed lipid-lowering efficacy of BMS both in vitro and in vivo. 


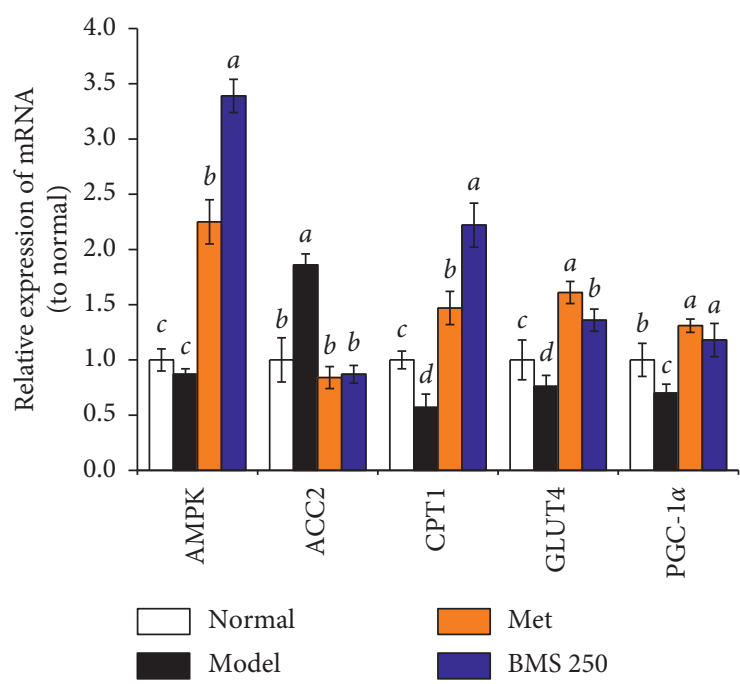

(a)

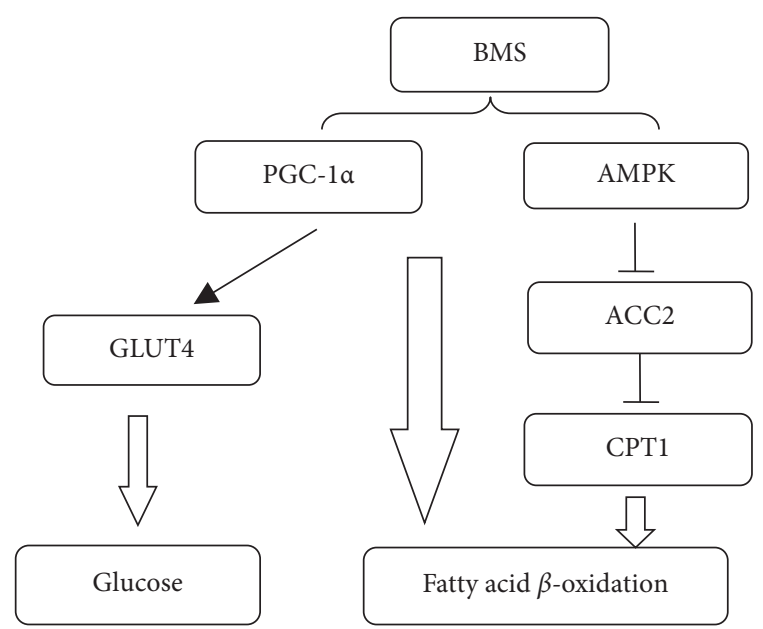

(b)

FIGURE 3: Effect of BMS on fatty acid $\beta$-oxidation and glucose intake. (a) mRNA expressions of AMPK, ACC2, CPT1, GLUT4, and PGC-1 $\alpha$ of HepG2-IR cells; (b) potential pathways of BMS involved in the glucose and lipid metabolism. Values are expressed as means \pm SE. Statistical analysis was performed using ANOVA. Different superscripts were considered significantly different. Met: metformin; BMS: bitter melon saponins.

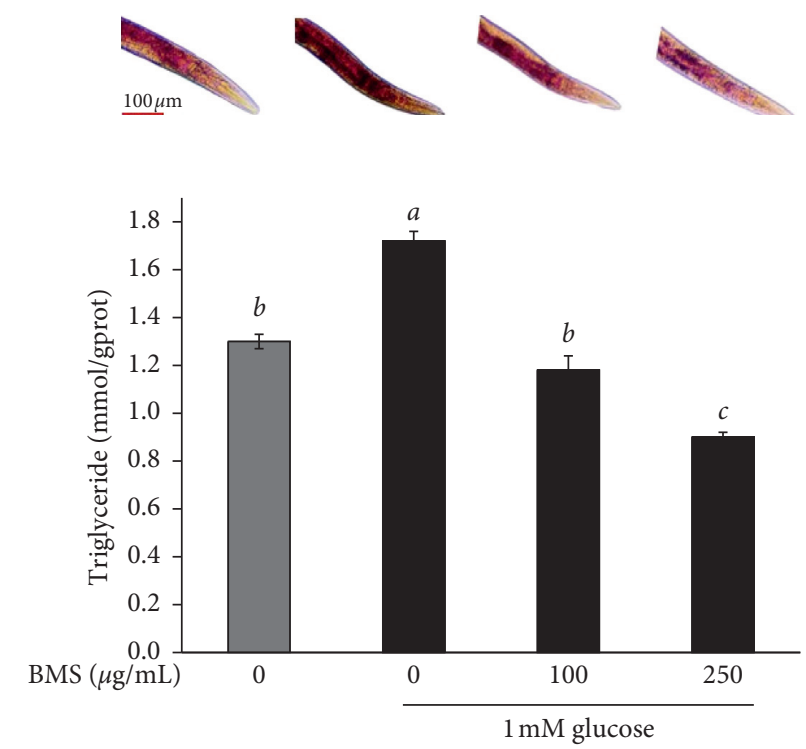

Figure 4: Effect of BMS on lipid accumulation (overall fat and TG content) in glucose-treated C. elegans. Values are expressed as means \pm SE. Statistical analysis was performed using ANOVA. Different superscripts were considered significantly different. BMS: bitter melon saponins.

3.5. Influences of BMS on the Locomotive Activity, Progeny Production, and Longevity in C. elegans. Genetic and environmental factors, including food, have been shown to affect the physical activities of $C$. elegans and could also play a large role in fat accumulation [21]. Thus, we needed to figure out whether BMS affected the basic growth and development of C. elegans. As shown in Figures 5(a) and 5(b), we found that BMS had no effect on the body bends of $C$. elegans, but it improved the frequency of head thrashes in glucose-treated worms, which inferred that BMS might slightly enhance the energy expenditure to lower the overall fat. Longevity of $C$. elegans is closely related with many factors, including environmental, dietary, and genetic factors [23, 24]. High concentration of glucose is shown to exhibit a negative impact on the lifespan of nematodes [25]. Regarding the progeny production and longevity, data suggested that BMS showed no influence on the brood size, while extending the median lifespan of glucose-treated worms, which indicated that both 100 and $250 \mu \mathrm{g} / \mathrm{mL}$ BMS exhibited no toxicity to the growth and development of C. elegans. 


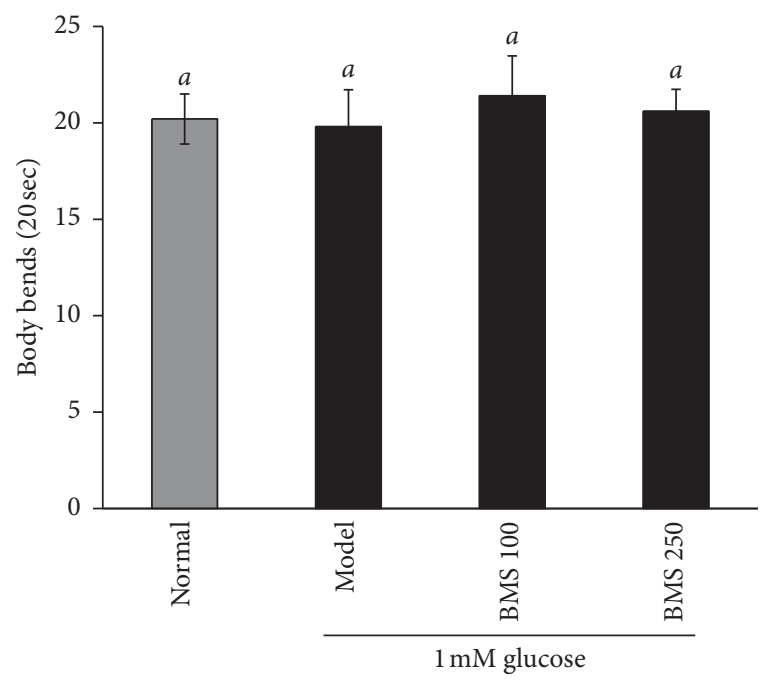

(a)

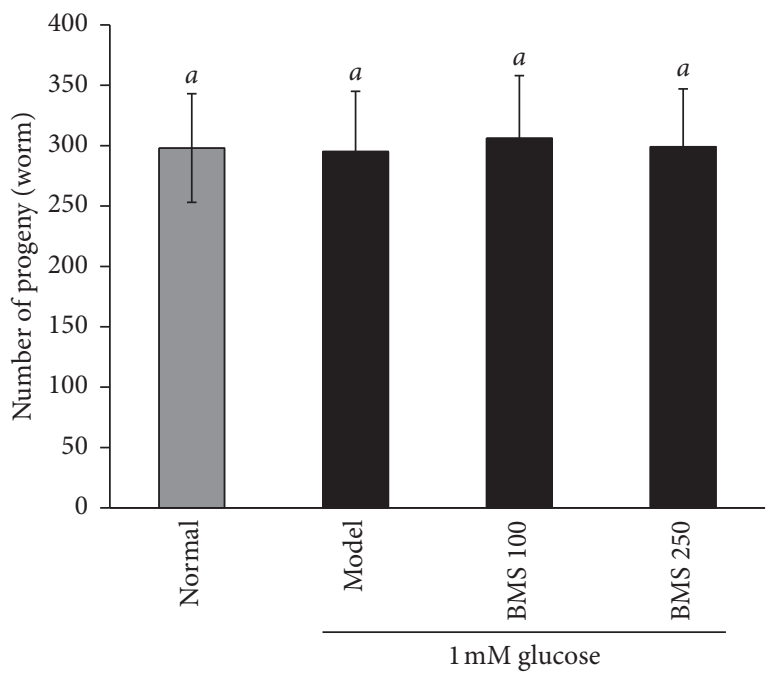

(c)

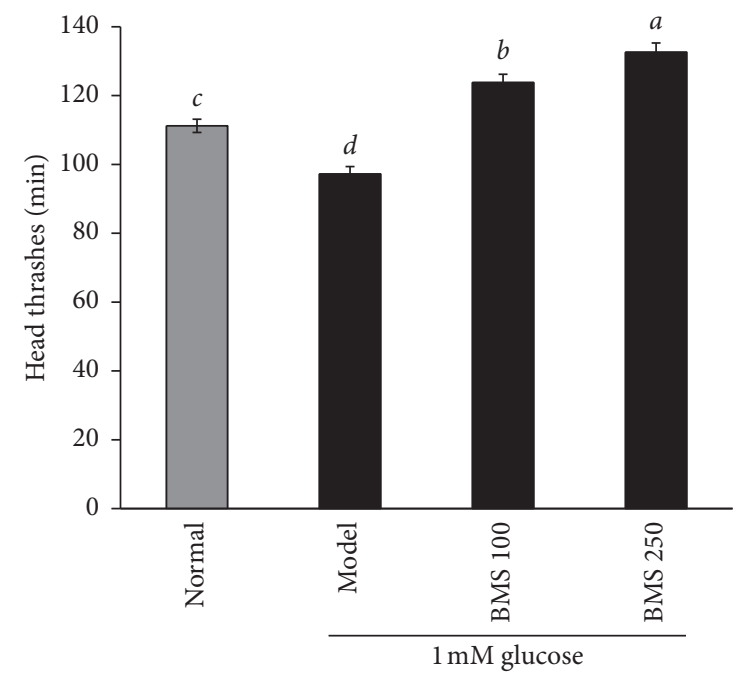

(b)

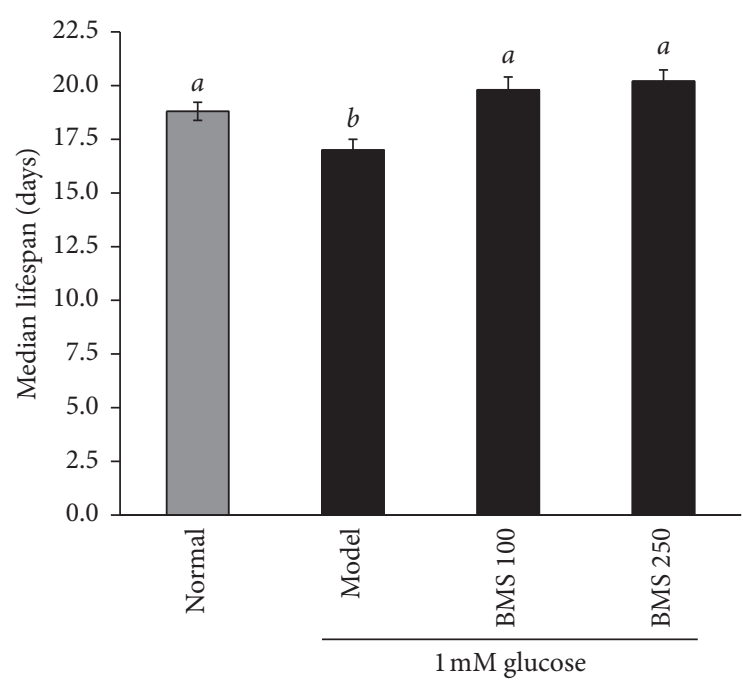

(d)

Figure 5: Effects of BMS on the locomotive activity, brood size, and lifespan of glucose-treated C elegans. (a) Body bends, (b) head thrashes, (c) progeny production, (d) median lifespan. Values are expressed as means \pm SE. Statistical analysis was performed using ANOVA. Different superscripts were considered significantly different. BMS: bitter melon saponins.

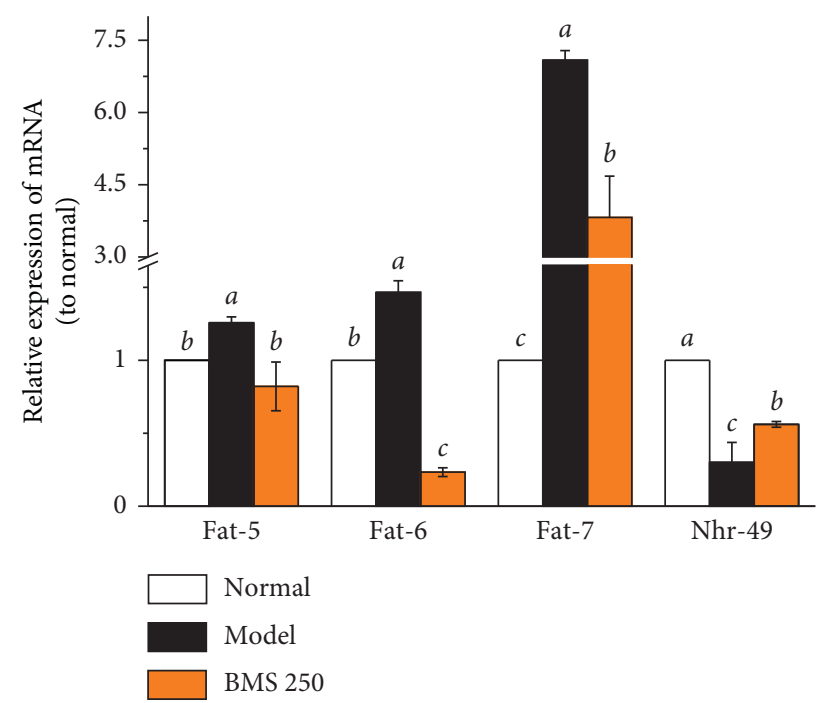

FIGURE 6: Effect of BMS on expressions of lipid metabolism related genes in C. elegans. Values are expressed as means \pm SE. Statistical analysis was performed using ANOVA. The means with different superscript were considered significantly different. BMS: bitter melon saponins. 
3.6. BMS Regulated the Expressions of Genes Involved in Lipid Metabolism in C. elegans. To discover the potential molecular pathways involved in the effect of BMS on fat reduction, we determined some important genes related lipid metabolism by qRT-PCR. In our current study, we found that BMS treatment profoundly decreased the mRNA expressions of desaturase genes, namely, fat- 1 , fat- 5 , and fat-7 (Figure 6), which are known as involved in the de novo synthesis of polyunsaturated fatty acids (PUFAs) [26]. Additionally, we tested a key gene expression involved fatty acid $\beta$-oxidation, the nuclear hormone receptor nhr-49 [27]. We found that $1 \mathrm{mM}$ glucose impaired the expression of nhr- 49 compared to that in normal group, while BMS significantly upregulated nhr-49 gene expression, indicating that BMS might act on nhr-49 to regulate catabolism of fatty acids by mediating the $\beta$-oxidation pathway, thereby reducing fat deposition in $C$. elegans. This was in accordance with the effects of BMS on enhancing $\beta$-oxidation in HepG2 cells.

\section{Conclusions}

In conclusion, BMS exerted beneficial effects on maintaining energy homeostasis, evidenced by enhancing glucose uptake and consumption in HepG2-IR model through upregulating GLUT4 expression and inhibiting fat accumulation in both HepG2 cell and $C$. elegans via promoting fatty acid $\beta$-oxidation. Prospectively, saponins from bitter melon might be a potential prebiotic to manage clinical patients with obesity or obesity-related metabolic syndrome.

\section{Data Availability}

All data generated or analyzed during this study are included in this article.

\section{Conflicts of Interest}

The authors declare that they have no conflicts of interest.

\section{Acknowledgments}

This research was supported by Jiangsu Post-Doctoral Research Funding Program (2020Z070), National Natural Science Foundation of China (31701598), and University Philosophy and Social Science Program (2019SJA1892).

\section{References}

[1] Y. C. Chooi, C. Ding, and F. Magkos, "The epidemiology of obesity," Metabolism, vol. 92, pp. 6-10, 2019.

[2] J. Bai, Y. Zhu, and Y. Dong, "Response of gut microbiota and inflammatory status to bitter melon (Momordica charantia L.) in high fat diet induced obese rats," Journal of Ethnopharmacology, vol. 194, pp. 717-726, 2016.

[3] J. Bai, Y. Zhu, and Y. Dong, "Modulation of gut microbiota and gut-generated metabolites by bitter melon results in improvement in the metabolic status in high fat diet-induced obese rats," Journal of Functional Foods, vol. 41, pp. 127-134, 2018.

[4] S. Wang, Z. Li, G. Yang, C.-T. Ho, and S. Li, "Momordica charantia: a popular health-promoting vegetable with multifunctionality," Food \& Function, vol. 8, no. 5, pp. 1749-1762, 2017.

[5] J. L. Perez, G. K. Jayaprakasha, and B. S. Patil, "Metabolite profiling and in vitro biological activities of two commercial bitter melon (Momordica charantia Linn.) cultivars," Food Chemistry, vol. 288, pp. 178-186, 2019.

[6] C. Y. Cheok, H. A. K. Salman, and R. Sulaiman, "Extraction and quantification of saponins: a review," Food Research International, vol. 59, pp. 16-40, 2014.

[7] D. G. Popovich, L. Li, and W. Zhang, "Bitter melon (Momordica charantia) triterpenoid extract reduces preadipocyte viability, lipid accumulation and adiponectin expression in 3T3-L1 cells," Food and Chemical Toxicology, vol. 48, no. 6, pp. 1619-1626, 2010.

[8] C.-C. Shih, C.-H. Lin, and W.-L. Lin, "Effects of Momordica charantia on insulin resistance and visceral obesity in mice on high-fat diet," Diabetes Research and Clinical Practice, vol. 81, no. 2, pp. 134-143, 2008.

[9] X. Xiao, X. Zhang, C. Zhang et al., "Toxicity and multigenerational effects of bisphenol S exposure to Caenorhabditis elegans on developmental, biochemical, reproductive and oxidative stress," Toxicology Research, vol. 8, no. 5, pp. 630-640, 2019.

[10] D. Gerlier and N. Thomasset, "Use of MTT colorimetric assay to measure cell activation," Journal of Immunological Methods, vol. 94, no. 1-2, pp. 57-63, 1986.

[11] G. M. Solis and M. Petrascheck, "Measuring Caenorhabditis elegans life span in 96 well microtiter plates," Journal of Visualized Experiments, no. 49, 2011.

[12] C. Kenyon, J. Chang, E. Gensch, A. Rudner, and R. Tabtiang, "A C. elegans mutant that lives twice as long as wild type," Nature, vol. 366, no. 6454, pp. 461-464, 1993.

[13] K. J. Livak and T. D. Schmittgen, "Analysis of relative gene expression data using real-time quantitative PCR and the 2- $\Delta \Delta$ CT method," Methods, vol. 25, no. 4, pp. 402-408, 2001.

[14] J. O. Lee, S. K. Lee, J. H. Kim et al., "Metformin regulates glucose transporter 4 (GLUT4) translocation through AMPactivated protein kinase (AMPK)-mediated cbl/CAP signaling in 3T3-L1 preadipocyte cells," Journal of Biological Chemistry, vol. 287, no. 53, pp. 44121-44129, 2012.

[15] M.-J. Tan, J.-M. Ye, N. Turner et al., "Antidiabetic activities of triterpenoids isolated from bitter melon associated with activation of the AMPK pathway," Chemistry \& Biology, vol. 15, no. 3, pp. 263-273, 2008.

[16] C. Kwon, J. L. Sun, J. H. Jeong, and T. W. Jung, "Humanin attenuates palmitate-induced hepatic lipid accumulation and insulin resistance via AMPK-mediated suppression of the mTOR pathway," Biochemical and Biophysical Research Communications, vol. 526, no. 2, pp. 539-545, 2020.

[17] B. Viollet, B. Guigas, N. S. Garcia, J. Leclerc, M. Foretz, and F. Andreelli, "Cellular and molecular mechanisms of metformin: an overview," Clinical Science, vol. 122, no. 6, pp. 253-270, 2012.

[18] S. Pang, D. A. Lynn, J. Y. Lo, J. Paek, and S. P. Curran, "SKN-1 and NRF2 couples proline catabolism with lipid metabolism during nutrient deprivation," Nature Communications, vol. 5, no. 1, p. 5048, 2014.

[19] Y. Peng, R. Y. Gan, H. B. Li et al., “Absorption, metabolism, and bioactivity of vitexin: recent advances in understanding the efficacy of an important nutraceutical," Critical Reviews in Food Science and Nutrition, vol. 16, pp. 1-16, 2020.

[20] J.-H. Han, N. Q. Tuan, M.-H. Park et al., "Cucurbitane triterpenoids from the fruits of momordica charantia improve insulin sensitivity and glucose homeostasis in streptozotocin- 
induced diabetic mice," Molecular Nutrition \& Food Research, vol. 62, no. 7, Article ID 1700769, 2018.

[21] X. Xiao, C. Tan, X. Sun et al., "Fermented barley $\beta$-glucan regulates fat deposition in Caenorhabditis elegans," Journal of the Science of Food and Agriculture, vol. 100, no. 8, pp. 3408-3417, 2020.

[22] L. R. Girard, T. J. Fiedler, T. W. Harris et al., "WormBook: the online review of Caenorhabditis elegans biology," Nucleic Acids Research, vol. 35, pp. D472-D475, 2007.

[23] T. He, L. He, E. Gao et al., "Fat deposition deficiency is critical for the high mortality of pre-weanling newborn piglets," Journal of Animal Science and Biotechnology, vol. 9, p. 66, 2018.

[24] J. Campisi, P. Kapahi, G. J. Lithgow et al., "From discoveries in ageing research to therapeutics for healthy ageing," Nature, vol. 571, no. 7764, pp. 183-192, 2019.

[25] D. J. Eisermann, U. Wenzel, and E. Fitzenberger, "Inhibition of chaperone-mediated autophagy prevents glucotoxicity in the Caenorhabditis elegans mev-1 mutant by activation of the proteasome," Biochemical and Biophysical Research Communications, vol. 484, no. 1, pp. 171-175, 2017.

[26] R. J. S. Reis, L. L. Xu, H. Lee et al., "Modulation of lipid biosynthesis contributes to stress resistance and longevity of C. elegans mutants," Aging-Us, vol. 3, no. 2, pp. 125-147, 2011.

[27] M. R. Van Gilst, H. Hadjivassiliou, and K. R. Yamamoto, "From the cover: a Caenorhabditis elegans nutrient response system partially dependent on nuclear receptor NHR-49," Proceedings of the National Academy of Sciences, vol. 102, no. 38, pp. 13496-13501, 2005. 